\title{
Age alone is not a barrier to concurrent chemoradiotherapy for advanced head and neck cancer
}

\author{
Gerard Sexton ${ }^{1}$, Paul Walsh² ${ }^{2}$ Frank Moriarty ${ }^{3}$, and James O’Neill ${ }^{1}$ \\ ${ }^{1}$ Beaumont Hospital \\ ${ }^{2}$ National Cancer Registry \\ ${ }^{3}$ Royal College of Surgeons in Ireland
}

May 17, 2021

\begin{abstract}
Objectives To evaluate the benefits imparted by concurrent chemoradiotherapy (CCRT) and chemotherapy of any form to elderly head and neck cancer (HNC) patients in Ireland. Secondary outcomes included comparison of these benefits to the adult population and subgroup analysis by site. Design, setting, and participants A retrospective cohort study was conducted using 20 years of cancer registry data provided by the National Cancer Registry of Ireland. All HNC diagnosed from 1994-2014 were included. Cox multivariate regression analysis was applied to test for the benefits of CCRT and chemotherapy of any form in HNC. The primary outcome measures were cancer-specific and all-cause survival in months. Results Survival analysis showed an overall benefit to the use of CCRT in patients with advanced disease over 65 years, particularly when used for hypopharyngeal, oral cavity, oropharyngeal, and laryngeal malignancy, though the latter did not achieve statistical significance. Chemotherapy of any form conferred a survival benefit in elderly patients with hypopharyngeal, laryngeal, nasopharyngeal, and oropharyngeal cancer. Conclusion CCRT and chemotherapy of any form confer significant survival benefits to appropriately selected elderly HNC patients and should therefore not be withheld solely on the basis of age.
\end{abstract}

\section{Hosted file}

HNC Paper CCRT blinded.pdf available at https://authorea.com/users/414161/articles/522281age-alone-is-not-a-barrier-to-concurrent-chemoradiotherapy-for-advanced-head-and-neckcancer 